\title{
Sample loading and retrieval by centrifugation in a closed-loop PCR microchip
}

\author{
Khoi Seng Lok, Yien Chian Kwok ${ }^{1}$, Nam-Trung Nguyen ${ }^{2}$ \\ ${ }^{1}$ National Institute of Education, Nanyang Technological University, 1 Nanyang \\ Walk, Singapore 637616, Singapore.E-mail: yienchian.kwok@nie.edu.sg \\ ${ }^{2}$ School of Mechanical and Aerospace Engineering, Nanyang Technological \\ University, 50 Nanyang Avenue, Singapore 639798.E-mail: mntnguyen@ntu.edu.sg.
}

\begin{abstract}
We report on a novel concept of sample loading for microfluidic devices using a ${ }_{10}$ benchtop centrifuge and a magnetically actuated circular closed-loop PCR microchip as a model system. The PCR mixture and the ferrofluid were loaded into a specially designed microchip. The microchip was then placed in an off-the-shelf 50-mL tube and centrifuged. The strong centrifugal force drives the PCR mixture and the ferrofluid into the microchannels of the microchip, and simultaneously expels any ${ }_{15}$ trapped microbubbles. PCR was successfully carried out on single and parallel closedloop PCR microchips. The addition of a few off-chip handling steps allows great simplification of the device design. This new loading concept may be useful for designing robust and low-cost lab-on-a-chip devices because benchtop centrifuges are quite common in most laboratories.
\end{abstract}

${ }_{20}$ Keywords: Lab-on-chip $\cdot$ Micro total analysis system $\cdot$ Centrifuge $\cdot$ PCR microchip Sample loading 


\section{Introduction}

Microfluidic devices deal with fluid flow in microchannels on a miniature platform.In lab-on-a-chip (LOC), laboratory protocols and procedures are implemented on a single microfluidic platform. Similarly, micro total analysis system ( $\mu$ TAS) is a concept of miniaturization of laboratory analysis onto a microfluidic platform [1-3]. In the development of such microfluidic platforms, sample loading is an important step which can be driven by electrokinetic force, air pressure and programmable syringe pumps. Qi et al. introduced a rapid and variable-volume sample loading in sieving electrophoresis microchips using negative pressure combined with electrokinetic force [4]. Zhang et al. used negative air pressure to pinch and inject the sample into an electrophoresis microchip[5]. Wang et al. used hydrostatic pressure for sample injection [6]. Programmable precision syringe pump can also be used to introduce liquids into microchannel via tubing. Dossi et al. used a hydrodynamic injection approach in a microchip for electrophoresis [7]. Among the many sample loading techniques that can be implemented in a microfluidic chip, centrifuge is a simple approach that has enough power to overcome the high pressure drop across microchannels.

Centrifugal force has been implemented on a LOC system in the form of a spinning compact disc (CD). This technology is also known as "lab-on-a-disc". The disc usually contains several diagnostic or functional units. In each unit, reagents and samples are placed near the centre of the disc. Capillary valves are used to control the fluid flow. Spinning this disc creates a centrifugal force that opens up these valves. The predeposited reagents and samples mix and react in the microchannel. Honda et al. used a commercial centrifugal platform (Gyrolab Bioaffy) to perform immunoassay for detection of biotinylated antibodies against $\alpha$-fetoprotein (AFP), interleukin-6 (IL-6), and carcinoembryonic antigen [8]. Grumann et al. adapted the CD-platform for batch-mode mixing, where magnetic force, alternated rotation were used to shorten the mixing time [9]. Riegger et al. also used such a platform for multiplexed, bead-based fluorescence assay for hepatitis A and tetanus [10]. Jia et al. used a CD-platform for DNA hybridization assay [11]. He et al. used this platform for cytokine enzyme-linked 
immunosorbent assay (ELISA) [12]. CD-platforms have many useful applications, but also have its own limitations. Centrifugal forces are uni-directional. The fluid is span outward to the edge of the disk. This fact limits the implementation of the centrifuge concept to certain types of assays. Kong et al. reported a solution for the problem of unidirectional pumping using a regulated stream of compressed gas to force the liquid radically toward the centre of the platform during the spinning process [13], but its implementation was complicated. In addition, CD-platform usually requires a weight balance. Once a sterile CD package is opened, all the diagnostic units have to be utilized immediately. The CD spinning system is also not a regular laboratory item and needs to be purchased to use with this unique LOC platform.

Polymerase chain reaction (PCR) is a technique to amplify nucleic acids (NAs) from a template containing a specific region of interest. PCR microchips are LOCs using PCR and its variants for detection of pathogens, biomarkers and DNA fingerprinting [14]. A PCR microchip was first introduced by Northrup et al. in 1993 [15]. Several reagents such as primers, polymerase, deoxynucleoside triphosphates (dNTPs), magnesium (II) ions and a NA template are needed to run PCR. The PCR mixture undergoes a series of thermal cycles involving denaturation, annealing, and extension/elongation. Conventional PCR is carried out in a thermocycler. Researchers have been trying various methods to implement this unique PCR protocol on a microfluidic chip [16]. PCR microdevices are implemented in stationary reaction chamber or flow-through channels. PCR are carried out in wells [17] or reaction chambers [18-20]. A heater changes the temperature temporally to achieve the effect of thermal cycling. In the flow-through concept, the PCR mixture is driven in the microchannel through different temperature zones to attain heating and cooling. The channels of the flow-through concept can be open or closed. Open designs have the serpentine [21-29] or spiral [30] forms. The number of PCR cycles is determined by the design and can not be changed. A closed-loop design for instance has the form of a circular channel, which allows running an unlimited number of PCR cycles.

West et al. designed a circular closed-loop PCR microchip that used magnetohydrodynamics to propel the PCR mixture [31]. However, the relatively high pumping 
speed of this concept limits the control of cycle duration. Sun et al. adopted the closedloop concept using magnetic actuation [31, 32]. An oil-based ferrofluid and the PCR mixture were injected into the microchip through a Teflon tube. The ferrofluid plug was actuated by the external magnetic field of a rotary permanent magnet. The oil-based plug was immiscible with the aqueous PCR mixture medium. Hence, the mixture was propelled by the plug along the microchannel. This concept was developed further to provide high-throughput using multiple parallel circular channels [33]. The PCR microchip design has some drawbacks. The ferrofluid is viscous and difficult to load in and remove from the microchip. The injection of the fluid introduces air bubbles into the channels. The trapped air bubbles disrupt the fluid flow and affect the PCR results. Due to the T-junction design, the channels are filled unevenly, when unequal pressure is applied. The above drawbacks could be overcome using a centrifugal force for sample loading reported in this paper.

The incorporation of the circular closed-loop PCR microchip into a conventional $\mathrm{CD}$ platform is challenging. The channel network needs to be redesigned, aligned and fabricated into the CD format. Since centrifugal force provides strong propulsion, loading sample onto the magnetically driven PCR microchip should be easy. A detachable design is preferred, because the device can be removed after sample loading and plugged into the PCR amplification system. As the centrifugal force is uni-directional, it is difficult to use the same force to retrieve the sample after the PCR run. Access to the sample needs to be included on the device. The design can be further simplified if the device can fit into a standard benchtop centrifuge for sample loading purpose.

In this report, we report a novel concept of sample loading and retrieval for microfluidic devices using a benchtop centrifuge, Fig. 1. The proof of concept is demonstrated using the magnetically actuated PCR microchip as a model. The PCR device is designed to fit into an off-the-shelf $50-\mathrm{mL}$ centrifuge tube. The samples are initially loaded onto the loading chamber of the device. The tube containing the device is centrifuged. The high centrifugal force loads the samples into the channels. This method allows loading fluids into fine microchannels, and simultaneously purging trapped air bubbles. This concept may be used for other microfluidic devices to facilitate pumping 
liquids, removing of micro-sized air bubbles, and separation in microchannels. Sample retrieval is achieved by reversing the orientation of the microchip in the tube.

\section{Materials and Methods}

\section{Conceptual Design}

The microchip is designed to fit into a regular $50-\mathrm{mL}$ centrifuge tube. The tube is usually sterile, $30 \mathrm{~mm}$ in diameter and $115 \mathrm{~mm}$ in length with a conical bottom and screwed cap. The sample fluid is loaded in the loading chamber of the microchip. This microchip is then inserted in this tube and centrifuged with a weight balance, Fig. 1(a). Depending on the arrangement of the chip in the tube, sample can be loaded into the PCR channel (left) or retrieved from there (right). Figure 1(b) shows the example of a PCR device with a single loop. The samples are loaded at one access port. The centrifugal force drives the fluid from the inlet filling the microchannel network of the device. The fluid will settle in channel, based on their density. Air is purged out from the microchannels, exiting by the other access port, Fig. 1(c). During sample retrieval, the centrifugal force assists the separation the fluids in the microchip. Ferrofluid, amplified PCR sample and oil are separated as layers in the loading chambers, Fig. 1(d).

Microchip design and fabrication

CorelDraw, designing software for vector graphics, was purchased from Corel (Canada, www.corel.com). A commercial CO2 laser system (Universal M-300 Laser Platform) was obtained from Universal Laser Systems Inc. (Arizona, USA, www.ulsinc.com). Polymethyl methacrylate (PMMA) sheet of 1-mm thickness was obtained from Ying Wang Acrylic Trading (Singapore). PMMA film of 0.05-mm thickness was obtained from Goodfellow (Huntingdon, England, www.goodfellow.com). Two types of circular closed-loop PCR microchips, single-loop and parallel-loop, were designed using CorelDraw. The designed pattern was then transferred to the laser machining system to make the parts. The parts were aligned and thermally bonded at $165^{\circ} \mathrm{C}$ under low pressure [34]. 
The single-loop microchip allows one sample to be amplified by PCR in a single run. Figure 1(b) depicts the design and dimensions of this device, which is assembled by bonding a 1-mm thick PMMA part to a 0.05-mm thin PMMA film as cover. The thin PMMA film reduces the temperature gradient between the heater and the PCR mixture. The final device measures $25 \mathrm{~mm}$ in width, $43 \mathrm{~mm}$ in height and $1.05 \mathrm{~mm}$ in thickness. The sample loading chamber measures $4 \mathrm{~mm}$ in width, $5 \mathrm{~mm}$ in height and $0.85 \mathrm{~mm}$ in thickness, resulting in a volume of approximately $17 \mu 1$. The microchannel connecting the loading chamber with the circular PCR channel, measures $0.2 \mathrm{~mm}$ in width, $0.3 \mathrm{~mm}$ in height and $14.8 \mathrm{~mm}$ in length. The circular channel has a diameter of $20 \mathrm{~mm}$, a width of $0.375 \mathrm{~mm}$, a height of $0.3 \mathrm{~mm}$, resulting in a volume of approximately $7 \mu \mathrm{L}$.

The parallel-loop PCR microchip allows four samples to be amplified simultaneously in a single run. The chip measures $25 \mathrm{~mm}$ in width, $43 \mathrm{~mm}$ in height and $2.05 \mathrm{~mm}$ in thickness. Figure 2 shows the schematic layout of the device. The threedimensional channel network is constructed by bonding two 1-mm thick PMMA parts to a $0.05-\mathrm{mm}$ thin PMMA film. The sample loading chamber of the microchip measures $2 \mathrm{~mm}$ in width, $5.55 \mathrm{~mm}$ in height and $1 \mathrm{~mm}$ in depth, resulting in a volume of $11.1 \mu \mathrm{L}$. An opening of $1 \times 2 \mathrm{~mm}$ is made on layer 1 to allow access to the loading chamber. Parafilm or glass slip can be used to cover these openings. Eight microchannels connecting the loading chambers with the circular PCR channelsare machined on the bottom side of layer 1. The PCR channels are machined on the bottom side of layer 2 . Eight via holes are drilled in layer 2 to provide channel connections from layer 1 to this layer. The PCR channels measure $0.2 \mathrm{~mm}$ in width, $0.3 \mathrm{~mm}$ in height and have varying lengths. In order to provide the same volume of about $7 \mu \mathrm{L}$, the concentric circular channels are designed to have varying width of $0.50,0.44,0.39$ and $0.36 \mathrm{~mm}$ and respective varying diameters of 15,17, 19 and $21 \mathrm{~mm}$. The heights of the channels are $0.3 \mathrm{~mm}$. The $0.05-\mathrm{mm}$ thin layer 3 seals the PCR channels and provides a close contact to the heater blocks.

Centrifuge test 
The PCR devices were first tested for simultaneous loading of oil-based ferrofluid and water-based sample. Oil-based ferrofluid (EMG 901), (dynamic viscosity of $\eta=8 \mathrm{mPa} \cdot \mathrm{s}$, density of $\rho=1.43 \times 10^{3} \mathrm{~kg} / \mathrm{m}^{3}$ ) were obtained from Ferrotec (Singapore, www.ferrotec.com.sg). A ferrofluid volume of $2 \mu \mathrm{L}$ and a volume of $5 \mu \mathrm{L}$ of deionised water were loaded into the chamber of the single-loop PCR microchip. Subsequently, PCR mixture was used in replacement of water. This microchip was put into the centrifuge tube, Fig. 1(a), and was centrifuged at varying speed from $500 \mathrm{rpm} / 48 \times g$ to the maximum setting of $4,200 \mathrm{rpm} / 3,392 \times g$, where $g=9.8 \mathrm{~m} / \mathrm{s}^{2}$ is the gravitational acceleration. Samples were retrieved by centrifuging again, as shown in Fig. 1(a) and (d).

PCR model

Lambda DNA template, bovine serum albumin (BSA), GoTaq@ Hot Start Green Master Mix, nuclease-free water and $1 \mathrm{~kb}$ DNA ladder was obtained from Promega (USA). GelRed $^{\mathrm{TM}}$ was obtained from Biotium (CA, ww.biotium.com). 50-mL centrifuge tubes were obtained from Greiner (Frickenhausen, Germany, www.greinerbioone.com). A centrifuge, Sigma 3-16 was purchased from Sartorius (Goettingen, Germany, www.sartorius.com). A thermocycler, GeneAmp PCR system 9700, was purchased from Life Technologies (USA, www.lifetechnologies.com). Gel image analysis software, Gel Analyzer, was downloaded from gelanalyzer.com.

There were two sets of primers used in this study: Set A and B. ${ }^{1}$ These primers were synthesized by 1st BASE (Singapore, www.base-asia.com). Primer set A were designed to select out 500 bp of DNA product for the lambda DNA template. Set B were designed to prime out $247 \mathrm{bp}$ of DNA product, which were within the region of interest of set A in the lambda DNA template. For each experiment conducted, a total of $100 \mu \mathrm{L}$ PCR master mixture was prepared in a 0.2-mL Eppendorf PCR tube. ThisPCR mixture was composed of $1 x$ GoTaq $@$ Hot Start Green Master Mix, $0.1 \mu \mathrm{M}$ of downstream and upstream primers each and $0.867 \mathrm{ng} \cdot \mu \mathrm{L}^{-1}$ of lambda DNA template and $1 \mu \mathrm{g} \cdot \mu \mathrm{L}^{-1}$ of BSA. A positive control of PCR amplification using primer set A was tested on the thermo cycler. ${ }^{2}$ For single-loop and parallel-loop PCR microchips, $2 \mu \mathrm{L}$ of ferrofluid, followed by $5 \mu \mathrm{L}$ of PCR mixture was loaded onto the chamber of the microchip. The ferrofluid 
had to cover one-third of the circular channel for effective pumping. The microchip was inserted upright into the $50 \mathrm{~mL}$ centrifuge test tube, Fig. 1(a). The tube containing the microchip was centrifuged at $3,000 \mathrm{rpm} / 1,730 \times \mathrm{g}$ to load the liquids into the PCR channel. Silicon oil or mineral oil was used to fill up the inlet chambers to prevent evaporation of water from the microchips.

Magnetically actuated PCR system

The prepared PCR microchips were then run using our PCR system reported previously [32-33]. The microchip was placed on the heater unit consisting of three blocks. The heater blocks provide the required temperature for denaturation, annealing and extension steps. Initially all three heater blocks were set at $95^{\circ} \mathrm{C}$ for $2 \mathrm{~min}$ to inactivate the antibody and to initiate the hot-start PCR. Subsequently, the heaters were set to $60^{\circ} \mathrm{C}$, $72{ }^{\circ} \mathrm{C}$ and $95^{\circ} \mathrm{C}$, respectively. A stepper motor moves the permanent magnet that in turn actuates the ferrofluid and the PCR mixture along the circular channel. The cycle time was set at $36 \mathrm{~s}$ per cycle. A total time of $15 \mathrm{~min}$ was needed to provide 25 cycles. PCR was carried out when the PCR mixture moved through the three temperature zones. Finally, all blocks were set to $72{ }^{\circ} \mathrm{C}$ for $3 \mathrm{~min}$ and cooled down to room temperature. The total run time was $20 \mathrm{~min}$.

To retrieve the amplified PCR sample, the single-loop PCR microchip was inserted into a clear $50 \mathrm{~mL}$ centrifuge tube so that the loading chambers are pointed outward, Fig. 1(a). The microchip was then centrifuged to remove the fluids from the channels. The sample will be emptied from the microchip and transfer to the bottom of the tube. A pipette was then used to remove this sample from the immiscible oil and ferrofluid. In the case of the parallel-loop PCR microchip, the silicon oil was removed by a pipette or a syringe. A transparent tape was used to cover the access holes. The microchip was inserted with the loading chamber in first into a 50-mL centrifuge tube and centrifuged. The centrifuge process returned the samples back to the loading chambers of microchips. A pipette was then used to retrieve the samples.

After the PCR run, the mixtures were analysed using 1.5\% agarose stack gel electrophoresis with gelRED ${ }^{\mathrm{TM}}$. The gel was then read using a UV transilluminator. 


\section{Results and discussions}

Single-looped microchip

\section{Sample loading}

To test out the amount of centrifugal force required to bring the fluid down into the circular channel of the microchip, the tube containing the microchip was centrifuged at varying speed from $500 \mathrm{rpm} / 48 \times g$ to the maximum setting of 4,200 rpm/3,392×g for a duration of $5 \mathrm{~min} .2 \mu \mathrm{L}$ of ferrofluid and $5 \mu \mathrm{L}$ of deionised water or PCR mixture was loaded onto the loading chamber of the microchip.The PCR mixture was prepared using the protocol as described earlier. This microchip was inserted in an upright position into a centrifuge tube. This tube containing the microchip was centrifuged in the speed, as described earlier. The microchip was taken out from the tube for comparison.

Figure 3(a) shows the ferrofluid and PCR mixture loaded onto the loading chamber of the microchip before it was centrifuged. Capillary action would draw the ferrofluid partially into the channel, but this was insufficient to fully load the fluid into the microchip. If the centrifugal speed was set from $500 \mathrm{rpm} / 48 \times g$ to $1,000 \mathrm{rpm} / 192 \times g$, no effective loading was observed. The fluids failed to reach the circular channel. At 1,500 rpm/437×g, partially loading of the samples was observed, Fig. 3(b). Trapped air bubbles can be found within the channels. These bubbles came from the poor pipetteing technique, in the initial loading phase when the sample was introduced onto the loading chamber. Empty channel was still observed in the circular channel. Above a speed of $1,500 \mathrm{rpm} / 437 \times g$, the force generated was sufficient to drive the liquids into the circular channel, Fig. 3(c). However, a high centrifugal force was needed to expel all the air bubbles. The ferrofluid with a higher specific density settled at the bottom of the microchip and automatically formed the magnetic plug. Above the layer of ferrofluid was the PCR mixture. The mixture spontaneously filled up the entire PCR channel. Air was expelled during this process. The result was a clear differentiated brown ferrofluid plug and green PCR mixture, with no air gaps in the circular channel. Hence, for subsequent experiments, the centrifuge speed was set at 3,000 rpm/1,730×g. 
As the volume of the circular channel was fixed, if more than a total volume of 7 $\mu \mathrm{L}$ was loaded into the microchip, excess fluid will appear in the loading chamber, Fig. 3(d). This excess fluid will not be participating in PCR amplification; this will not affect the overall performance of the microchip system.

\section{Sample retrieval}

To retrieve the sample from the microchip, the microchip was inserted in an inverted position into a clean the 50-mL centrifuge tube. The centrifugal speed was set at 3,000 $\mathrm{rpm} / 1,730 \times g$. At such a strong centrifugal force, all the samples would be expelled from the microchip and dropped into the bottom of the tube. Figure 3(e) shows the unloaded sample in the centrifuge tube after it was centrifuged. The circular channel of the microchip was clear and emptied. The ferrofluid, PCR mixture, and silicon oil were found at the bottom of the tube. The denser ferrofluid settles as the bottom layer, followed by the green PCR mixture. Silicone oil stays on top of the PCR mixture. Both ferrofluid and silicon oil were immiscible with the water-based PCR mixture. The tube containing the amplified samples may be stored for further experiments. The PCR mixture can be retrieved later by a regular pipette or syringe. Although $5 \mu 1$ of PCR mixture was introduced into microchip, only 4.5 to $4.8 \mu \mathrm{L}$ of its fluid was retrieved after the PCR run. This 4 to $10 \%$ loss of fluid was probably due to the poor pipette technique, evaporation of water or minor absorption of fluid into the immiscible phase. Nevertheless, this remaining PCR product was sufficient to produce a robust intense DNA band in gel electrophoresis later.

Parallel-loops microchip

\section{Sample loading}

To investigate if sample loading was of equivalent efficiency in the parallel-loops microchip as in the previous single-loop microchip, a similar centrifuge test was conducted. Four samples were loaded individually at the four loading chambers on the left of the microchip. The microchip was inserted into the centrifuge tube and was being centrifuged at 3,000 $\mathrm{rpm} / 1,730 \times g$. 
The results were similar to the single-loop microchip, as described earlier. Figure 4(a) shows the loaded samples after the tube containing the microchip was centrifuged. The brown denser ferrofluid settled at the lower portion of the circular channels, forming the desired ferrofluid plug. The PCR mixture completely filled up the rest of the circular channels, leaving no air gaps in between. The microchip was well fabricated with no leakage in between the channels. A clear distinction of fluids in each concentric circular channel was observed, with no leakage into the neighboring channels.

Fabrications of multiple layered microchip was extremely challenging. The three dimension channel network was connected by via holes through each layer, as mentioned earlier. Precise alignment was needed as the gap in-between channels were $0.5 \mathrm{~mm}$. A misalignment in the PMMA parts will result in fluid leakage. Under high centrifugal force, the fluid may be forced from its channel into the neighboring channel. Owning to good alignment of the PMMA parts and the "high heat and low pressure" thermobonding method [34], fabrication of this microchip was made possible.

\section{Sample retrieval}

Sample retrieval from the parallel-loops microchip was challenging and not as direct as single-loop microchip. Careful consideration was needed to ensure that the neighbouring samples were not contaminated one and another. The silicon oil was removed to create room for the sample fluid to return to the loading chambers. A transparent tape was used to seal the access opening of this microchip.

Figure 4(b) shows the retrieval of the four samples after the microchip was centrifuged. ${ }^{3}$ The ferrofluid will be on the top layer, followed by the amplified PCR sample. The distributions of the sample fluids for each loop were uneven. The circular channels were clear and emptied. The samples were removed by pipetteing from both labelled openings of the microchip, which contained the same sample. The successful retrieved amount was from 4.0 to $4.5 \mu \mathrm{L}$ after the PCR run. This low retrieval amount was due to its complication, being distributed at both openings of the microchip; an extra step was needed to remove one portion and combined with the other. This step was needed as $2.5 \mu \mathrm{l}$ of sample would be insufficient to give an intense band in the later gel 
electrophoresis. The parallel-loops microchip proposed some inconveniences in sample retrieval, in traded for being able to run the maximum of four samples in a single PCR run. In future work, design can implement to allow the samples to flow back to one single loading chamber instead of two openings, this should solve this issue.

Compare to parallel-loops microchip, the single-loop microchip is easier to fabricate and use using this centrifugal concept. Although, for both microchip designs both offers straightforward sample loading, the retrieval of samples from the parallelloops microchip is more difficult. It is important to design the microchannel network in the microchip is such a manner to prevent air bubbles from trapping inside. For instance, $\mathrm{W}$-shaped channel may accumulate undesirable air pocket in the middle while this problem is less obvious in the V-shaped channel. ${ }^{4}$ The unidirectional centrifugal force is only able to move the fluid in a vertical or slanted channel but not around $\mathrm{U}$-shaped bent or horizontal channel. In the current work if the inlet channels are defined to be too far apart, the central region of the circular channel cannot be filled up by fluid. In future designs, special considerations are needed to avoid microstructures that may create air pockets. For example, in the staggered herringbone micromixer [5], the indentation in the channel wall may also create air pockets in the microchip. An air vent is also required to allow fluid to enter and air to exit. Hence the centrifugal approach has its own drawbacks. As this work focuses on establishing the conceptual implementation of centrifugal technique, more work can be done to characterize this method quantitatively.

The sample loading by centrifuge makes the loading of samples onto the PCR microchips easier. The right volume of fluid can be directed to fill up the PCR channels. The ferrofluid plug and the PCR mixture can be separated quickly by the different densities of the liquids. The previously published designs [32,33] requires the injection of the liquids through small access holes on the microchip. This process introduces air bubbles and is laborious. Skilled hands are needed fluids in the microlitres scale. The previous designs pose technical challenges for untrained laboratory workers to use such a microchip. The current designs allow much simpler handling and only require basic pipetteing skills to use this microchip. If air bubbles do get into the circular channel, they may be purged out easily from the microchip by this technique. 


\section{PCR runs}

Several PCR runs were carried out to test the microchips using a magnetically actuated PCR system. Table 1 listed these test runs. The samples are loaded onto and retrieved from the microchips by the centrifugal method. The samples were analysed by gel electrophoresis. Figure 5 shows the UV gel image. In PCR run A, expected 247-bp and 500-bp DNA product was observed. In run B, equal intensity of 500-bp DNA bands were observed in all the concentric circular channels, showing that the amplifications were of similar effectiveness in each sample. In run $\mathrm{C}$, different samples were loaded to test for leakage or contamination in the parallel-loops microchip. No DNA product was observed in loop 1, because no primer was added. The 247-bp and 500-bp products were present in loop 2 and 3 respectively proving that DNA amplification was successfully carried out. In loop 4, an unexpected 300-bp and expected 500-bp product was observed. Since primer set $B$ selected out the region of interest within set A, there might be another preference region within these upstream and downstream primers. Perhaps, it was not suitable to mix set $\mathrm{A}$ and $\mathrm{B}$ together under the current PCR conditions. Despite this problem, PCR amplification was still successful in loop 4.

Sample loading using centrifugal technique in the new designs of PCR microchips enhances the DNA amplification system. This new concept speeds up the loading time and prevents trapped air bubbles from disrupting the fluid flow. 50-mL centrifuge tubes and benchtop centrifuges are widely available in laboratories. If it is not available, a simple eggbeater centrifuge may also be used to spin such a microchip for use in remote locations [36]. A square microchip can be designed to be flipped in different direction in the test tube and repeatedly centrifuged to achieve more complex functions. Smaller microchips can be designed to fit into a $15-\mathrm{mL}$ test tube and centrifuge in a similar manner. Capillary valves can be used to better control the fluid flow in these devices. High centrifugal force allows viscous liquids to be driven in microchannels. This concept is applicable to most protocols previously implemented on lab-on-a-disk platforms. Each diagnostic unit can be implemented as a modular stand-alone microchip. Combining the modules allows complex functions such as batch-mode mixing of reagents [9], fluorescence immunoassay [10], DNA hybridization [11], cytokine enzyme linked 
immunosorbent assay [12], and fast colorimetric alcohol assay from a single droplet of whole blood [38].

\section{Conclusions}

Sample loading by centrifugal force using a benchtop centrifuge is an innovative concept for loading and retrieval of samples in microfluidic devices. Lab-on-a-chip devices can be designed to fit into standard $15-\mathrm{ml}$ or $50-\mathrm{ml}$ laboratory test tubes. High centrifugal force is able to force a viscous fluid through the microchannels and to load it into a very fine channels and chambers. Trapped air bubbles in the channel are primed out in the same process. Using the circular closed-loop PCR microchip as a model, this method allows the arrangement of the ferrofluid below the PCR mixture and readily forms the driving magnetic plug for the device. DNA amplifications were successfully conducted using the magnetically actuated circular closed-loop PCR system. 


\section{References}

1. Vilkner T, Janasek D, Manz A (2004) Micro total analysis systems. Recent developments. Anal Chem 76(12):3373-3386. doi:10.1021/ac040063q

2. West J, Becker M, Tombrink S, Manz A (2008) Micro Total Analysis Systems: Latest Achievements. Anal Chem 80 (12):4403-4419. doi:10.1021/ac800680j

3. Dittrich PS, Tachikawa K, Manz A (2006) Micro total analysis systems. Latest advancements and trends. Anal Chem 78 (12):3887-3908. doi:10.1021/ac0605602

4. Qi LY, Yin XF, Zhang L, Wang M (2008) Rapid and variablevolume sample loading in sieving electrophoresis microchips using negative pressure combined with electrokinetic force. Lab on a Chip 8(7):1137-1144

5. Zhang LYX, Fang Z (2006) Negative pressure pinched sample injection for microchipbased electrophoresis. Lab Chip 6:258-264. doi:10.1039/b511924c

6. Wang W, Zhou F, Wu W (2009) A micro-electrophoresis system based on a short capillary with hydrostatic pressure assisted separation and injection. Microchimica Acta 166(1):35-39. doi:10.1007/s00604-009-0160-4

7. Dossi N, Toniolo R, Susmel S, Pizzariello A, Bontempelli G (2010) A simple approach to the hydrodynamic injection in microchip electrophoresis with electrochemical detection. Electrophoresis 31(15):2541-2547. doi:10.1002/elps.201000089

8. Honda N, Lindberg U, Andersson P, Hoffmann S, Takei H (2005) Simultaneous Multiple Immunoassays in a Compact Disc-Shaped Microfluidic Device Based on Centrifugal Force. Clin Chem 51 (10):1955-1961. doi:10.1373/clinchem.2005.053348

9. Grumann M, Geipel A, Riegger L, Zengerle R, Ducree J (2005) Batch-mode mixing on centrifugal microfluidic platforms. Lab Chip 5(5):560-565

10. Riegger L, Grumann M, Nann T, Riegler J, Ehlert O, Bessler W, Mittenbuehler K, Urban G, Pastewka L, Brenner T, Zengerle R, Ducrée J (2006) Read-out concepts 
for multiplexed bead-based fluorescence immunoassays on centrifugal microfluidic platforms. Sensors and Actuators A: Physical 126(2):455-462

11. Jia G, Ma K-S, Kim J, Zoval JV, Peytavi R, Bergeron MG, Madou MJ (2006) Dynamic automated DNA hybridization on a CD (compact disc) fluidic platform. Sensors and Actuators B: Chemical 114 (1):173-181

12. He H, Yuan Y, Wang W, Chiou N-R, Epstein AJ, Lee J (2009) Design and testing of a microfluidic biochip for cytokine enzymelinked immunosorbent assay. Biomicrofluidics 3(2):022401

13. Kong MCR, Salin ED (2010) Pneumatically Pumping Fluids Radially Inward On Centrifugal Microfluidic Platforms in Motion. Anal Chem. doi:10.1021/ac102071b

14. Sun Y, Kwok YC (2006) Polymeric microfluidic system for DNA analysis. Anal Chim Acta 556(1):80-96

15. Northrup M, Ching M, White R, Watson R (1993) DNA amplification with a microfabricated reaction chamber. Transducers

16. Zhang C, Xu J, Ma W, Zheng W (2006) PCR microfluidic devices for DNA amplification. Biotechnol Adv 24(3):243-284. doi: S0734-9750(05)00125-4

17. Khandurina J, McKnight TE, Jacobson SC, Waters LC, Foote RS, Ramsey JM (2000) Integrated System for Rapid PCR-Based DNA Analysis in Microfluidic Devices. Anal Chem 72(13):2995-3000. doi:10.1021/ac991471a

18. Neuzil P, Pipper J, Hsieh T (2006) Disposable real-time microPCR device: lab-on-achip at a low cost. Mol Biosyst Jun 2(6-7):292-298

19. Guttenberg Z, Müller H, Habermüller H, Geisbauer A, Pipper J, Felbel J, Kielpinski M, Scriba J, Wixforth M (2005) Planar chip device for PCR and hybridization with surface acoustic wave pump. Lab Chip 5:308-317 
20. Kim H, Vishniakou S, Faris GW (2009) Petri dish PCR: laserheated reactions in nanoliter droplet arrays. Lab Chip 9:1230-1235. doi:10.1039/b817288a

21. Schneegaß I, Bräutigam R, Köhler JM (2001) Miniaturized flowthrough PCR with different template types in a silicon chip thermocycler. Lab Chip 1(42-29). doi:10.1039/b103846j

22. Sun K, Yamaguchi A, Ishida Y, Matsuo S, Misawa H (2002) A heater-integrated transparent microchannel chip for continuousflow PCR. Sensors and Actuators $84: 283-289$

23. Liu Y, Ganser D, Schneider A, Liu R, Grodzinski P, Kroutchinina N (2001) Microfabricated polycarbonate $\mathrm{CE}$ devices for DNA analysis. Anal Chem 73(17):4196-4201

24. Li S, Fozdar DY, Ali MF, Li H, Shao D, Vykoukal DM, Vykoukal J, Floriano PN, Olsen M, McDevitt JT, Gascoyne PRC, Chen S (2006) A continuous-flow polymerase chain reaction microchip with regional velocity control. Microelectromechanical Systems 15(1):223-236

25. Hashimoto M, Barany F, Xu F, Soper SA (2007) Serial processing of biological reactions using flow-through microfluidic devices: coupled PCR/LDR for the detection of low-abundant DNA point mutations. Analyst 132(9):913-921. doi:10.1039/b700071e

26. Nakayama T, Kurosawa Y, Furui S, Kerman K, Kobayashi M, Rao SR, Yonezawa Y, Nakano K, Hino A, Takamura Y, Tamiya E (2006) Circumventing the air-bubbles for microfluidic systems and quantitative continuous-flow PCR applications. Anal Bioanal Chem 386:1327-1333

27. Crews N, Wittwer C, Gale B (2008) Continuous-flow thermal gradient PCR. Biomedical Microdevices 10(2):187-195. doi:10.1007/s10544-007-9124-9

28. Obeid PJ, Christopoulos TK, Crabtree HJ, Backhouse CJ (2003) Microfabricated Device for DNA and RNA Amplification by Continuous-Flow Polymerase Chain 
Reaction and Reverse Transcription-Polymerase Chain Reaction with Cycle Number Selection. Anal Chem 75(2):288-295. doi:10.1021/ac0260239

29. Obeid PJ, Christopoulos TK (2003) Continuous-flow DNA and RNA amplification chip combined with laser-induced fluorescence detection. Analytica Chimica 494:19

30. Hashimoto M, Chen PC, Mitchell MW, Nikitopoulos DE, Soper SA, Murphy MC (2004) Rapid PCR in a continuous flow device. Lab Chip 4:638-645. doi:10.1039/b406860b

31. West J, Karamata B, Lillis B, Gleeson J, Alderman J, Collins J, Lane W, Mathewson A, Berney H (2002) Application of magnetohydrodynamic actuation to continuous flow chemistry. Lab Chip Nov 2(4):224-230

32. Sun Y, Kwok YC, Nguyen NT (2007) A circular ferrofluid driven microchip for rapid polymerase chain reaction. Lab Chip 7 (8):1012-1017

33. Sun Y, Nguyen NT, Kwok YC (2008) High-throughput polymerase chain reaction in parallel circular loops using magnetic actuation. Anal Chem 80(15):6127-6130. doi:10.1021/ac800787g

34. Sun Y, Kwok YC, Nguyen N-T (2006) Low-pressure, hightemperature thermal bonding of polymeric microfluidic devices and their applications for electrophoretic separation. J Micromech Microeng 16

35. Stroock AD, Dertinger SKW, AjdariA, Mezic I, Stone HA, Whitesides GM (2002) Chaotic mixer for microchannels. Science 295:647-651

36. Wong AP, Gupta M, Shevkoplyas SS, Whitesides GM (2008) Egg beater as centrifuge: isolating human blood plasma from whole blood in resource-poor settings. Lab on a Chip 8(12):2032-2037 
37. Wei M-Y, Guo L-H, Famouri P (2011) DNA biosensors based on metallo-intercalator probes and electrocatalytic amplification.Microchimica Acta 172(3):247-260. doi:10.1007/s00604-010-0519-6

38. Steigert J, Grumann M, Brenner T, Riegger L, Harter J, Zengerle R, Ducree J (2006) Fully integrated whole blood testing by realtime absorption measurement on a centrifugal platform. Lab Chip 6(8):1040-1044 


\section{List of Tables}

Table 1 Summarises the PCR run conducted in this study. 


\section{List of Figures}

Fig.1 Sample loading by centrifuge: (a) Schematic illustration of the microchip in a 50-mL centrifuge tube and during the loading and retrieval process; (b) Design layout of the PCR chip with a single loop; Schematic illustration of the fluid flow in the microchip during the centrifuge process for (c) loading and (d) retrieval. F is the centrifugal force.

Fig. 2 Layout of the PCR microchip with four loops for four different samples.

Fig. 3 Photograph of the single-loop PCR chip (a)loaded before centrifuged, (b)partially loaded sample after centrifuged at 1,500 rpm/437×g, (c) loaded after centrifuged, (d) excess loading of fluid after centrifuged, and (e) unloaded sample in 50-mL centrifuge tube with microchip after centrifuged.

Fig. 4 Photograph of the parallel-loop PCR microchip, (a) loaded samples after centrifuged and (b) retrieval of the four samples after centrifuged.

Fig. 5 UV image of PCR products analyzed by gel electrophoresis. Set A is PCR run using single loop PCR microchip. Lane 1: 1kb DNA ladder; Lane 2: 500 bp PCR product from a commercial thermocycler as a control.Lanes 3: 247 bp PCR product; Lane 4: 500 bp PCR product. Set B and C are PCR runs using parallel-loop PCR microchip. Lane 5-8, 500 bp PCR products from loop 1 to 4 respectively. Lane 9-12: negative control (no primers), 247, 500, 300/500 bp PCR products from loop 1 to 4 respectively. 
Table 1

\begin{tabular}{lllll}
\hline PCR run & Microchip & Loop & Primer set & $\begin{array}{l}\text { Expected } \\
\text { product (bp) }\end{array}$ \\
\hline A1 & Single & 1 & B & 247 \\
A2 & Single & 2 & A & 500 \\
B & Parallel & 1 & A & 500 \\
& & 2 & A & 500 \\
& & 3 & A & 500 \\
C & & 4 & A & 500 \\
& & 1 & No primer & None \\
& & 2 & B & 247 \\
& & 3 & A & 500 \\
& & 4 & A \& B & $247 / 500$ \\
\hline
\end{tabular}



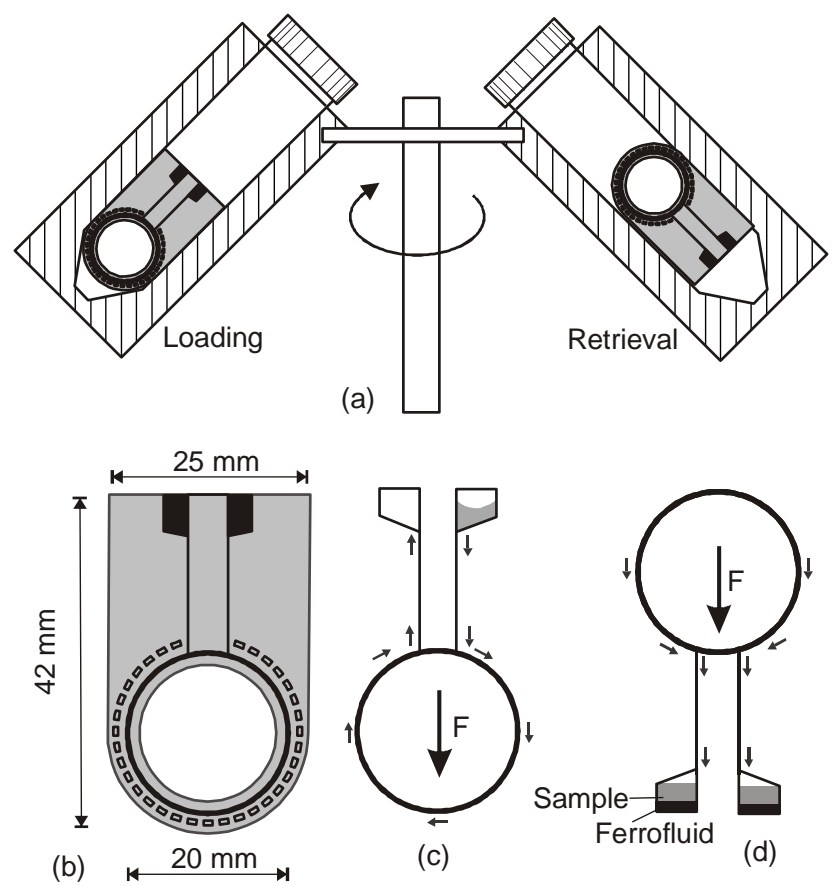

Fig.1 


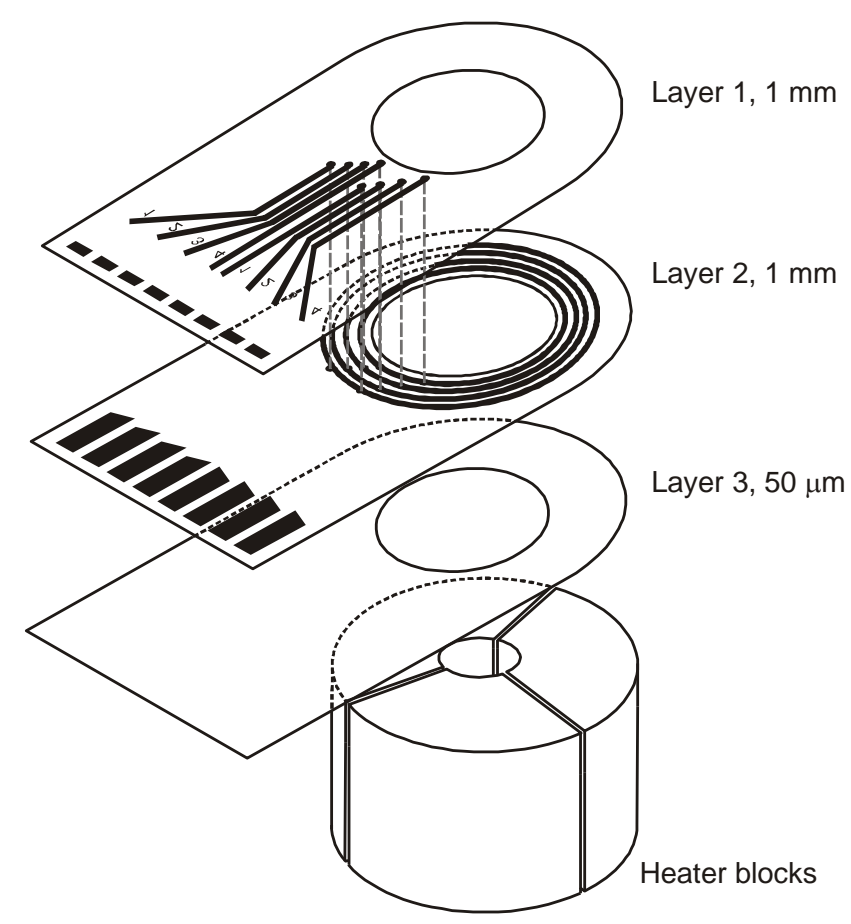

Fig. 2 


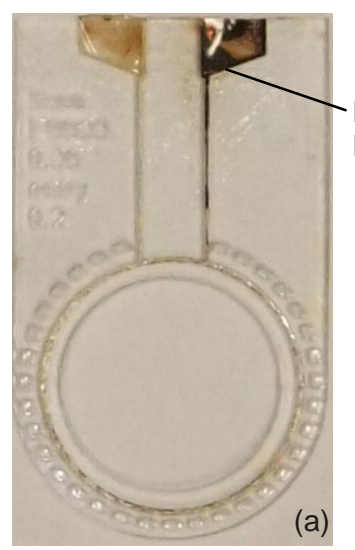

Ferrofluid \&

PCR mixture

(a)

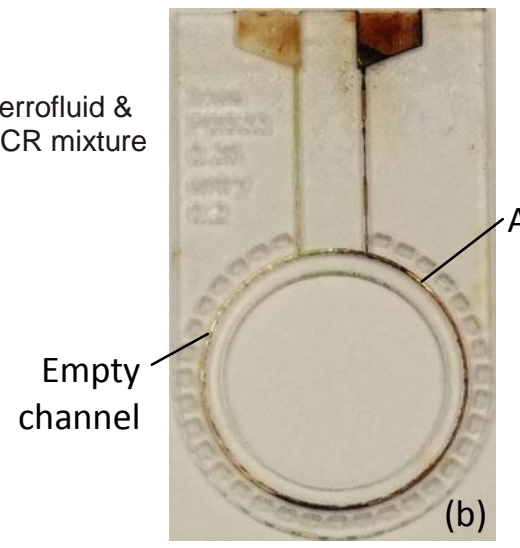

Air bubbles
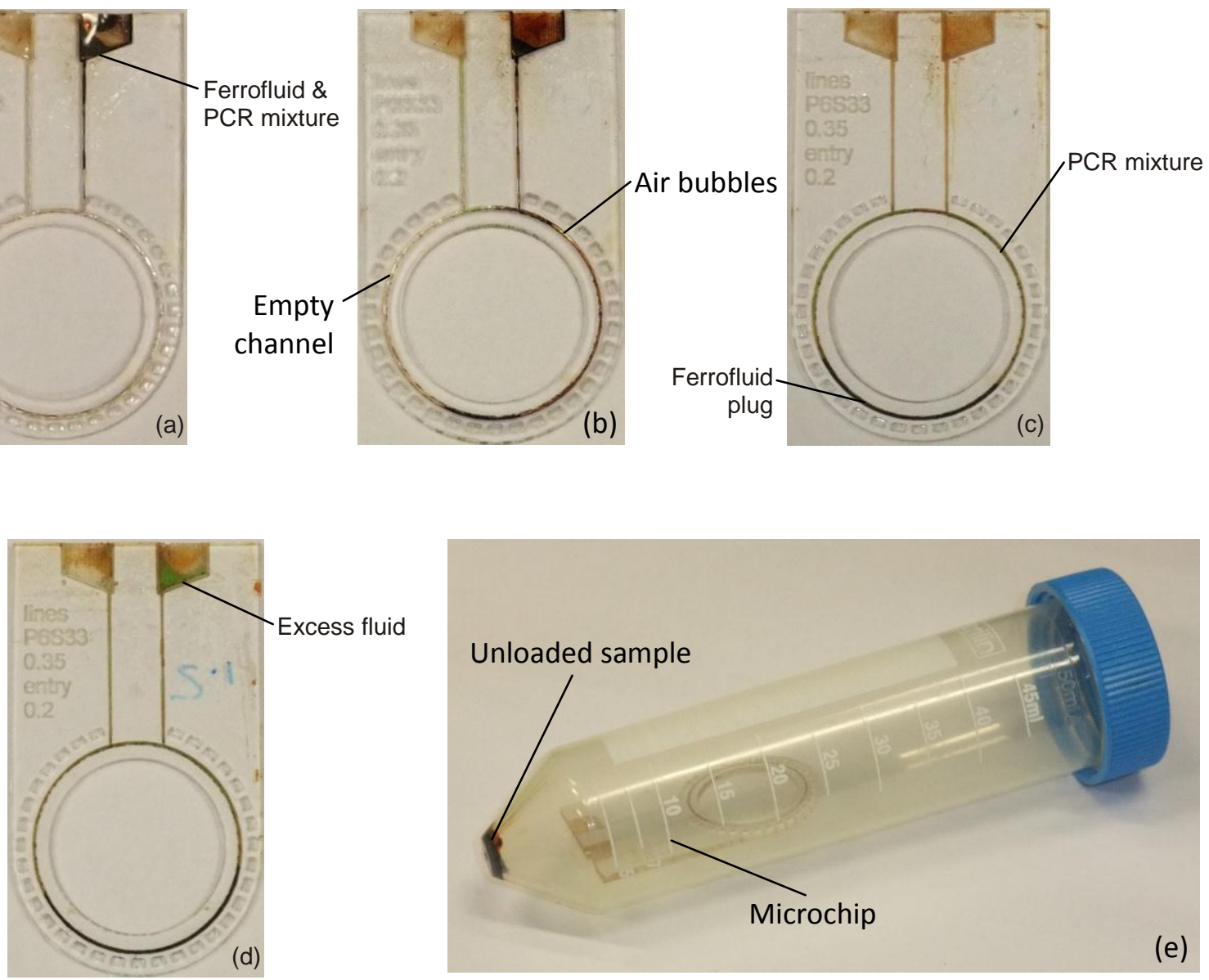

Fig. 3 

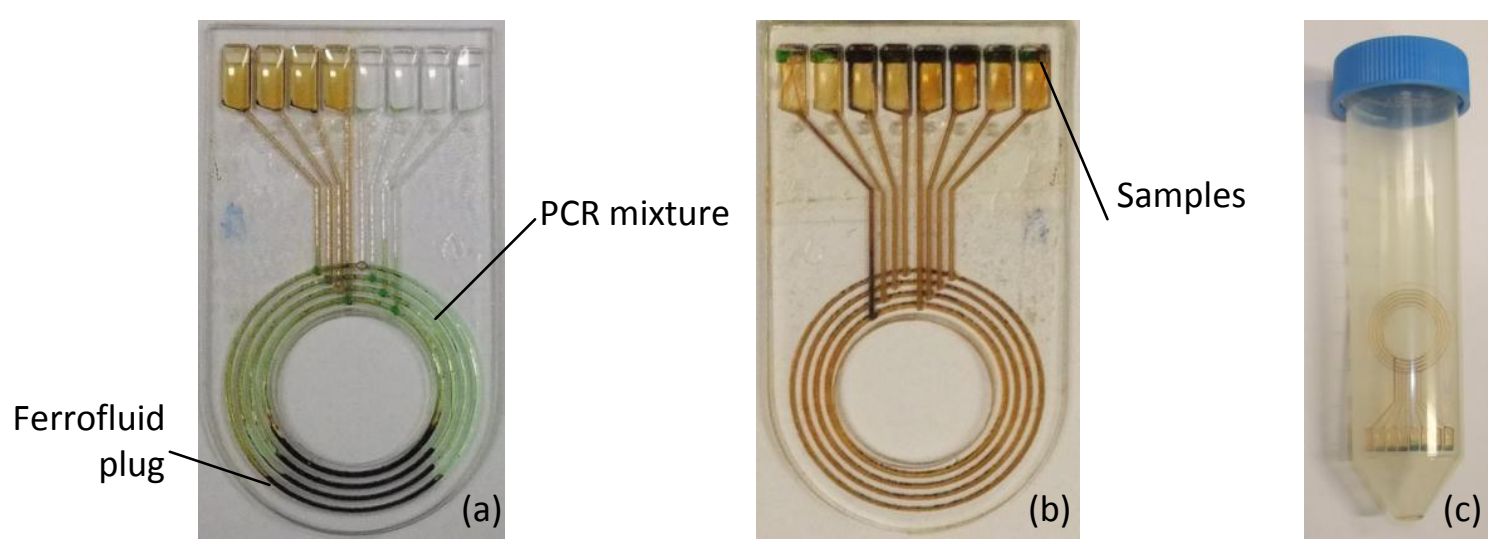

Fig. 4 


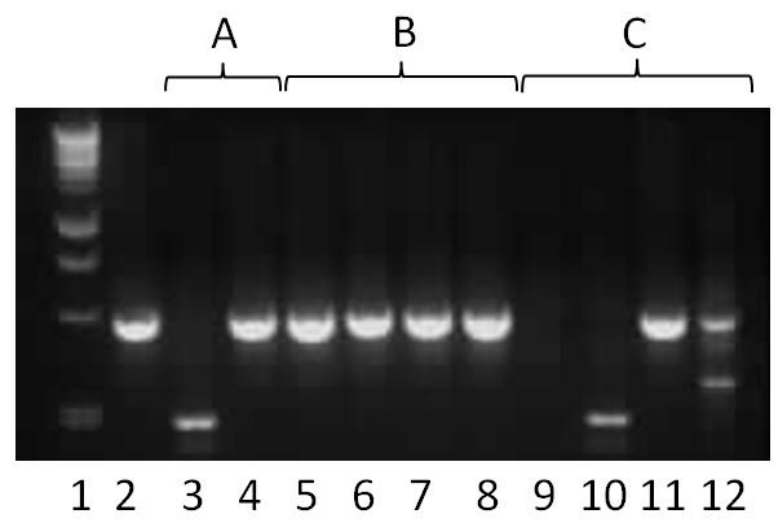

Fig.5 